UCRL-ID-128434

\title{
Methodology for the Relative Risk Assessment in the LDF Safety Analysis Report
}

\author{
S. J. Brereton
}

September 3, 1997

This is an informal report intended primarily for internal or limited external distribution. The opinions and conclu sions stated are those of the author and may or may not be those of the Laboratory.

Work performed under the auspices of the U.S. Department of Energy by the Lawrence Livermore National Laboratory under Contract W-7405-Eng-48. 


\section{DISCLAMER}

This document was prepared as an scoount of work sponsored by an agency of the United States Government. Neither the United States Government nor the University of California nor any of their employees, makes any warranty, express or implied, or assumes any legal liability or responsibility for the accuracy, completeness, or usefulness of any information, apparatus, product, or process disclosed, or represents that its use would not infringe privately owned rights. Reference herein to any specific commercial product, process, or service by tride name, trademark, manufacturer, or otherwise, does not necessarily constitute or imply its endorsement, recommendation, or favoring by the United States Government or the University of California. The views and optnions of authors expressed herein do not necessarlly state or reflect those of the United States Government or the University of California, and shall not be used for advertising or product endorsement purposes.

This report has been reproduced directly from the best available copy.

Available to DOE and DOE contractors from the Office of Scientific and Technical Information

P.O. Box 62, Oak Ridge, TN 37831

Prices available from (615) 576-8401, FTS 626-8401

Available to the public from the

National Technical Information Service

U.S. Department of Commerce

5285 Port Royal Rd.

Springfield, VA 22161 


\section{Methodology for the Relative Risk Assessment in the LDF Safety Analysis Report}

\subsection{Summary}

This document provides the methodology used for the relative risk assessment performed in the LDF Safety Analysis Report. The safety analysis for a facility of the hazard level of the LDF Complex (Buildings 490L, 492 are low hazard) should be mostly qualitative. This was the approach taken for the LDF risk assessment, where qualitative descriptors were assigned to event consequences and frequencies. The event consequences and frequencies were then combined using a risk matrix to obtain an assessment of the relative risk presented by each event to LDF workers and to the public. The development of the risk matrices is the main subject of this report. The matrices have been applied in the LDF SAR (LLNL, 1997).

\subsection{Overview of Methodology For Risk Matrix Development}

Two risk matrices are developed here: a chemical risk matrix for workers, and a chemical risk matrix for the public. Two matrices were needed because the consequence criteria used in establishing the matrices are different for workers and the public. Each is a $4 \times 4$ matrix, with consequences on the vertical axis and frequency on the horizontal axis.

In order to determine the relative risk presented by each of the 16 blocks of either of the risk matrices, numerical values must be assigned. This is accomplished by providing numerical values to the frequency and consequence axes. The product of the numerical values of frequency and consequence gives a range of numerical values defining the risk for each block. The numerical values for risk are compared and binned into risk ranges, allowing the relative risk of each of the 16 blocks of the matrix to be known. For simplicity and conservatism, the binning is based on the highest numerical value associated with a risk block (as opposed to using the range of values).

The basis for the numerical values for frequencies and consequences is given below. This is followed by the approach used for binning into risk ranges. The risk matrices are provided at the end of the document. 


\subsection{Frequency Levels}

All of the matrices have the same four frequency categories: Frequency Category 1, Frequency Category 2, Frequency Category 3, and Frequency Category 4. Numerical ranges were assigned to each category as given below. These ranges are commonly used for frequency binning, although the name given to a particular category may vary (DOE, 1994; DOE 1989a).

Erequency Category 1:

Condition exists or is expected at the facility $\left(\mathrm{f}>10^{-1} \mathrm{yr}^{-1}\right)$.

Erequency Category 2:

Incidents that may occur several times during the lifetime of the facility $\left(10^{-1} \mathrm{yr}^{-1}>\mathrm{f}>10^{-2} \mathrm{yr}^{-1}\right)$.

Erequency Category 3:

Accidents that are not anticipated to occur during the lifetime of the facility $\left(10^{-2} \mathrm{yr}^{-1}>\mathrm{f}>10^{-4} \mathrm{yr}^{-1}\right)$.

Erequency Category 4:

Accidents that will probably not occur during the life cycle of the facility $\left(10^{-4} \mathrm{yr}^{-1}>\mathrm{f}>10^{-6} \mathrm{yr}^{-1}\right)$.

\section{Beyond Erequency Category 4:}

All other accidents (facility $\left(\mathrm{f}<10^{-6} \mathrm{yr}^{-1}\right.$ ).

The probability of occurrence is so small that a reasonable scenario is not conceivable. These events are not considered in the facility design or safety analysis.

\subsection{Consequence Levels}

The consequence categories are provided below. They were selected to cover the range of potential impacts to workers or the public. The chemical consequences were selected on the basis of acute health effects 1 .

Consequence Category 1:

There is essentially no noticeable impact.

LDF Workers: exposure to chemical concentration less than TLV-TWA ${ }^{2}$

Public: exposure to chemical concentration less than one-tenth the TLVTWA

Consequence Category 2:

The impact is minor, reporting may be required.

LDF Workers: exposure to chemical concentration between TLV-TWA and ERPG-23 or equivalent; worker injury

\footnotetext{
${ }^{1}$ None of the chemicals considered in the LDF risk assessment were carcinogens. If this methodology were to be applied to another situation, where carcinogens were of interest, the methodology should be reviewed to ensure that these types of effects are appropriately included.

${ }^{2}$ TLV-TWA: Threshold Limit Value, Time Weighted Average, is the maximum concentration level of hazardous material to which a worker can be exposed for 8 hours per day, $\mathbf{4 0}$ hours per week.
} 
Public: exposure to chemical concentration between one-tenth the TLVTWA and ERPG-1 14 or equivalent

Consequence Category 3:

The impact is significant.

LDF Workers: exposure to chemical concentration between ERPG-2 or equivalent and ERPG-35 or equivalent; lost-time injury, chemical exposure causing significant discomfort or which is somewhat disabling Public: exposure to chemical concentration between ERPG-1 or equivalent and ERPG-2 or equivalent, i.e. measurable chemical exposure

\section{Consequence Category 4:}

The impact is severe.

LDF Workers: exposure to chemical concentration above ERPG-3 or equivalent i.e. death, disability, potential severe short-term health effects. Public: exposure to chemical concentration between ERPG-2 or equivalent and ERPG-3 or equivalent.

The consequence criteria require further development, as the determination of relative risk within the matrices requires numerical values. The consequence scale should be expressed in terms reflecting the relative magnitude of harm or injury that would be associated with exposure to a certain chemical concentration. In some cases, the numerical values of ERPG-2 and ERPG-3 concentrations are different by less than a factor of two; in other cases, they may differ by an order of magnitude. But, the magnitude of harm or injury associated with any of the ERPG concentrations is defined, and the same definitions apply for all chemicals. Thus, it is more appropriate to define a scale for chemical consequences representing the level of harm from an exposure, rather than the concentration to which one was exposed.

In order to define a scale, some level of subjectivity will be involved. Values of harm or consequence should be related to a reference point. For example, the harm associated with exposure to the TLV-TWA concentration could be set equal to one. If it is assumed that below the TLV, the consequence associated with exposure is linear, then exposure to a concentration of one-tenth the TLV-TWA would give a value for the consequence scale of 0.1 .

At the ERPG-1 level, one may experience some mild adverse health effects or perceive an objectionable odor. This is clearly a more significant consequence when compared to an exposure to the TLV-TWA, where continuous exposure for

3ERPG-2: Emergency Response Planning Guide, Level 2, as defined by the American Industrial Hygiene Association, is the maximum airborne concentration level of hazardous material below which it is believed that nearly all individuals could be exposed for up to one hour without experiencing or developing irreversible or other serious health effects or symptoms that could impair their ability to take protective action.

${ }^{4}$ ERPG-1: Emergency Response Planning Guide, Level 1, as defined by the American Industrial Hygiene Association, is the maximum airborne concentration level of hazardous material below which it is believed that nearly all individuals could be exposed for up to one hour without experiencing other than mild transient adverse health effects or perceiving a clearly defined objectionable odor.

5ERPG-3: Emergency Response Planning Guide, Level 3, as defined by the American Industrial Hygiene Association, is the maximum airborne concentration level of hazardous material below which it is believed that nearly all individuals could be exposed for up to one hour without experiencing or developing life threatening effects. 
$40 \mathrm{~h}$ a week would not result in any health effects. Since this is only an approximate methodology for ranking events relative to one another, it would be reasonable to assign the health effects associated with exposure at the ERPG-1 level a value of 10 .

At the ERPG-2 level, one may experience reversible health effects. This consequence was judged to be 10 times more severe than the consequences one might experience at exposure to the ERPG-1 level. The value assigned to the ERPG-2 health effects on the consequence scale would then be 100 (relative to the TLV-TWA reference point, which is assigned a value of 1 ).

At the ERPG-3 level, one may experience irreversible health effects. Because these health effects could include permanent disability, this was thought to be considerably more severe than exposure at the ERPG-2 level. Thus, the ERPG-3 consequences were assigned a value 100 times greater than the ERPG-2 consequences. On the consequence scale, ERPG-3 consequences would then have a value of 10,000. Another way to look at this is in terms of a trade-off. It was thought to be reasonable to consider the exposure of 100 individuals (either workers or members of the public) to the ERPG-2 concentration, resulting in temporary but significant effects, equal to the exposure of 1 individual (also in either population) to the ERPG-3 concentration, which could result in permanent effects.

The last consequence of interest is death, and this was utilized only for the development of the worker risk matrix. Again, this health effect was thought to be significantly more severe than the previous level, and the consequences were assigned a value 100 times greater than the ERPG-3 consequences. On the consequence scale, this results in a numerical value of $10^{6}$ for death. In terms of a trade-off, it seemed reasonable that exposure of 100 workers to the ERPG-3 concentration, potentially resulting in permanently disabling effects, was approximately equal to the exposure of 1 worker to a lethal concentration, which would result in death.

The numerical values for the consequence scale are summarized below:

\section{Chemical Consequence Category 1:}

LDF Workers: exposure to chemical concentration less than TLV-TWA; in relative terms, less than 1

Public: exposure to chemical concentration less than one-tenth the TLVTWA; in relative terms, less than 0.1

\section{Chemical Consequence Category 2:}

LDF Workers: exposure to chemical concentration between TLV-TWA and ERPG-2 or equivalent; in relative terms, between 1 and 100

Public: exposure to chemical concentration between one-tenth the TLVTWA and ERPG-1 or equivalent; in relative terms, between 0.1 and 10

Chemical Consequence Category 3:

LDF Workers: exposure to chemical concentration between ERPG-2 or equivalent and ERPG-3 or equivalent; in relative terms, between 100 and $10^{4}$

Public: exposure to chemical concentration between ERPG-1 or equivalent and ERPG-2 or equivalent; in relative terms, between 10 and 100 
Chemical Consequence Category 4:

LDF Workers: exposure to chemical concentration above ERPG-3 or equivalent, up to and including death; in relative terms, between $10^{4}$ and $10^{6}$

Public: exposure to chemical concentration between ERPG-2 or equivalent and ERPG-3 or equivalent; in relative terms, between 100 and $10^{4}$.

\subsection{Assignment of Risk Ranges}

Risk is the product of frequency and consequence. For each block in the two risk matrices the corresponding numerical values for frequencies and consequences were multiplied, providing a numerical value for the risk. Since the frequencies and consequences span a range of values, the risk will also span a range of values. To simplify the process, and to be conservative, the highest values of frequency and consequence corresponding to a given block were used to determine a value for risk. These values are indicated on Figures 1 and 2.

As an example, for worker events (Figure 1), Consequence Category 2 ranges from 1 to 100 , and Frequency Category 2 ranges from $10^{-2}$ to $10^{-1} / \mathrm{yr}$. The numerical values for risk for the block on Figure 1 corresponding to Consequence Category 2 and Frequency Category 2 would range from $10^{-2}$ to 10 . For simplicity and conservatism, the highest value, 10, was used in the risk binning, and this value is shown on the figure. This process was similarly applied to give the numerical values on all of the blocks in Figures 1 and 2.

The next step was to identify blocks of the matrices, which represent similar risk. This was done by establishing five risk bins, representing five risk levels. Five risk levels were thought to be adequate to allow for enough distinction between events on a $4 \times 4$ matrix. Also, there were three reasonable reference points from which one could make risk level assignments, and this lead to a total of five risk levels These were identified as Risk Levels 1 through 5, with Risk Level 1 representing the lowest risk, and Risk Level 5 representing the highest risk.

Three premises were identified on which the binning and Risk Level selection were based:

(1) The risk associated with an event in Frequency Category 1 and resulting in the upper bound Category 1 consequence to the public would be the upper bound for risk in Level 1.

(2) The risk associated with an event in Frequency Category 1 and resulting in the upper bound Category 1 consequence to workers would be the upper bound for risk in Level 2.

(2) The risk associated with an event in Frequency Category 2 (event occurring sometime during the life of the facility) resulting in the death of a worker (i.e. upper bound for Category 4 consequence for workers) would be the lower bound for risk in Level 5. 
Risk levels 3 and 4 were selected to fit evenly between risk levels 2 and 5.

Figures 1 and 2 present the risk matrices, and numerical values associated with each risk block for chemical risk to workers and to the public.

An upper bound for Risk Level 1 can be obtained from premise 1 noted above. This would correspond to the highest numerical value of risk associated with a Category 1 consequence to the public from a Frequency Category 1 occurrence. The highest numerical value associated with a Category 1 consequence to the public is 0.1 (corresponding to a concentration equal to one-tenth the TLV-TWA). The highest frequency associated with a Frequency Category 1 event is $1\left(\mathrm{yr}^{-1}\right)$. Thus, the numerical value for the risk would be 0.1 , and Risk Level 1 would include all blocks on Figures 1 and 2 with numerical values of risk less than or equal to 0.1 .

An upper bound for Risk Level 2 can be obtained from premise 2 noted above. This would correspond to the highest numerical value of risk associated with a Category 1 consequence to workers from a Frequency Category 1 occurrence. The highest numerical value associated with a Category 1 consequence to workers is 1 (corresponding to a concentration equal to the TLV-TWA). The highest frequency associated with a Frequency Category 1 event is $1\left(\mathrm{yr}^{-1}\right)$. Thus, the numerical value for the risk would be 1, and Risk Level 2 would include all blocks on Figures 1 and 2 with numerical values of risk between 0.1 and 1 .

A lower bound for Risk Level 5 can be obtained from premise 3 noted above. In this case, the value would correspond to the least likely event that could occur during the life of a facility (i.e. Frequency Category 2 event) that could result in death to a worker. The low end of the Frequency Category 2 range is $10^{-2}\left(\mathrm{yr}^{-1}\right)$. The numerical value associated with death was selected at $10^{6}$. The product of frequency and consequence gives a numerical value of $10^{4}$. Thus, Risk Level 5 would include all blocks on Figures 1 and 2 with numerical values of risk greater than $10^{4}$.

The other two risk ranges were assigned to fit between these bounds as noted below:

Risk Level 1: risk value $\leq 0.1$

Risk Level 2: $0.1<$ risk value $\leq 1$

Risk Level 3: $1<$ risk value $\leq 100$

Risk Level 4: $100<$ risk value $\leq 10^{4}$

Risk Level 5: risk value $>10^{4}$

\subsection{Assignment of Relative Risk}

The numerical values on each risk matrix (obtained by multiplying the appropriate consequence value and frequency value) were compared to the risk range values given in the previous section. This allowed each of the risk blocks to be assigned a Risk Level. In each of the two figures, blocks of similar risk level were shaded the same. The shading of each block will allow one to determine if an event placed in that risk block presents more or less risk than an event in another risk block. 
Some observations can be made regarding the risk matrices. It is apparent that the region of low risk (Risk Level 1) has expanded for the public, while that of Level 5 risk does not exist, and that of Risk Level 4 has become smaller when compared to the worker matrix. This is a direct result of the lower consequence values assigned to the public consequence categories. Because the numerical consequence criteria are lower for the public, the risk value for a given block will be lower. Since the risk binning process uses the same numerical values for workers and the public, more blocks from the public risk matrix will fall into the lower risk levels. When placing the blocks of a risk matrix into risk bins, the workers and the public are viewed the same, i.e. the same criteria are used. In effect, this normalizes the risk to both populations.

The figures indicate (by similar shading of the risk blocks that are being compared) that a Frequency Category 3 event resulting in a Category 4 consequence to a worker (including death) presents similar risk to a Frequency Category 1 or 2 event resulting in a Category 4 consequence to the public. Or, a Frequency Category 4 event resulting in a Category 4 consequence to a worker (including death) presents similar risk to a Frequency Category 1 or 2 event occurring with a Category 3 consequence to the public.

\subsection{Application of Risk Matrices}

To make use of the risk matrices, it is first necessary to identify events that will or might occur at the facility. This is accomplished through a detailed hazards analysis. Each event should then be assigned frequency and consequence descriptors. For most events, there will be two consequences descriptors: one for the public and one for workers. In some cases, a given event may affect only one population. It is not necessary to quantitatively evaluate a frequency and consequence for each event. These only need to be known well enough to place an event into a category (i.e. known within a range of values). The criteria for the frequency and consequence categories given here are to be used as guidelines. The frequency and consequences associated with an event, can be based on judgment, or estimated from existing documentation on the facility of interest or other similar facilities.

Once frequencies and consequences have been assigned to all events identified for the facility, the relative risk associated with each event can be determined. Each event should be reviewed and placed in the appropriate block of the relevant matrix or matrices (two matrices would be used if an event can affect both workers and the public). The relative risk is determined from the shading of the block where the event is placed.

Once the relative risk of all events identified for a facility is known, those presenting higher risk can be studied in more detail. Events presenting relatively higher risk would be readily identified by this process and might be candidates for risk reduction. 


\subsection{References}

DOE (1989a), "Safety Analysis and Review System", San Francisco Operations Office Management Directive, SAN MD 5481.1A, U.S. Department of Energy, June 1989.

DOE (1994), "Preparation Guide for U.S. Department of Energy Nonreactor Nuclear Facility Safety Analysis Reports, DOE Standard, DOE-STD-3009-94, U.S. Department of Energy, July 1994.

LLNL (1997), "Safety Analysis Report for the Laser Demonstration Facility Complex", August 1997.

\section{Acknowledgement}

The author would like to acknowledge and thank Tom Altenbach for the interesting and useful input and discussions on risk assessment. 


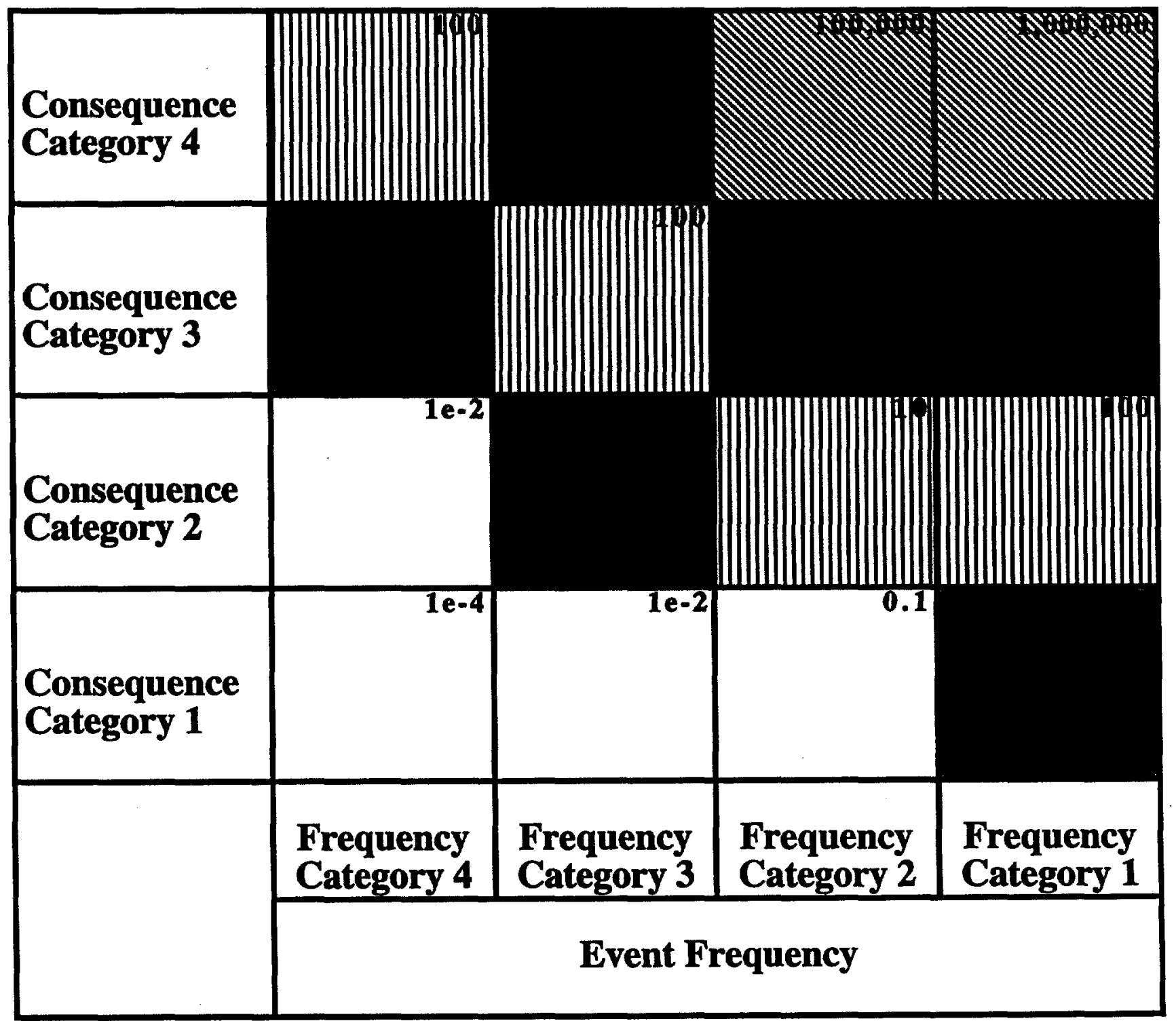

\begin{tabular}{|c|c|}
\hline & Risk Level 1 \\
\hline & Risk Level 2 \\
\hline & Risk Level 3 \\
\hline & Risk Level 4 \\
\hline & Risk Level 5 \\
\hline
\end{tabular}

Figure 1: $\quad$ Risk Matrix for relative ranking of worker chemical release events for the LDF Complex 


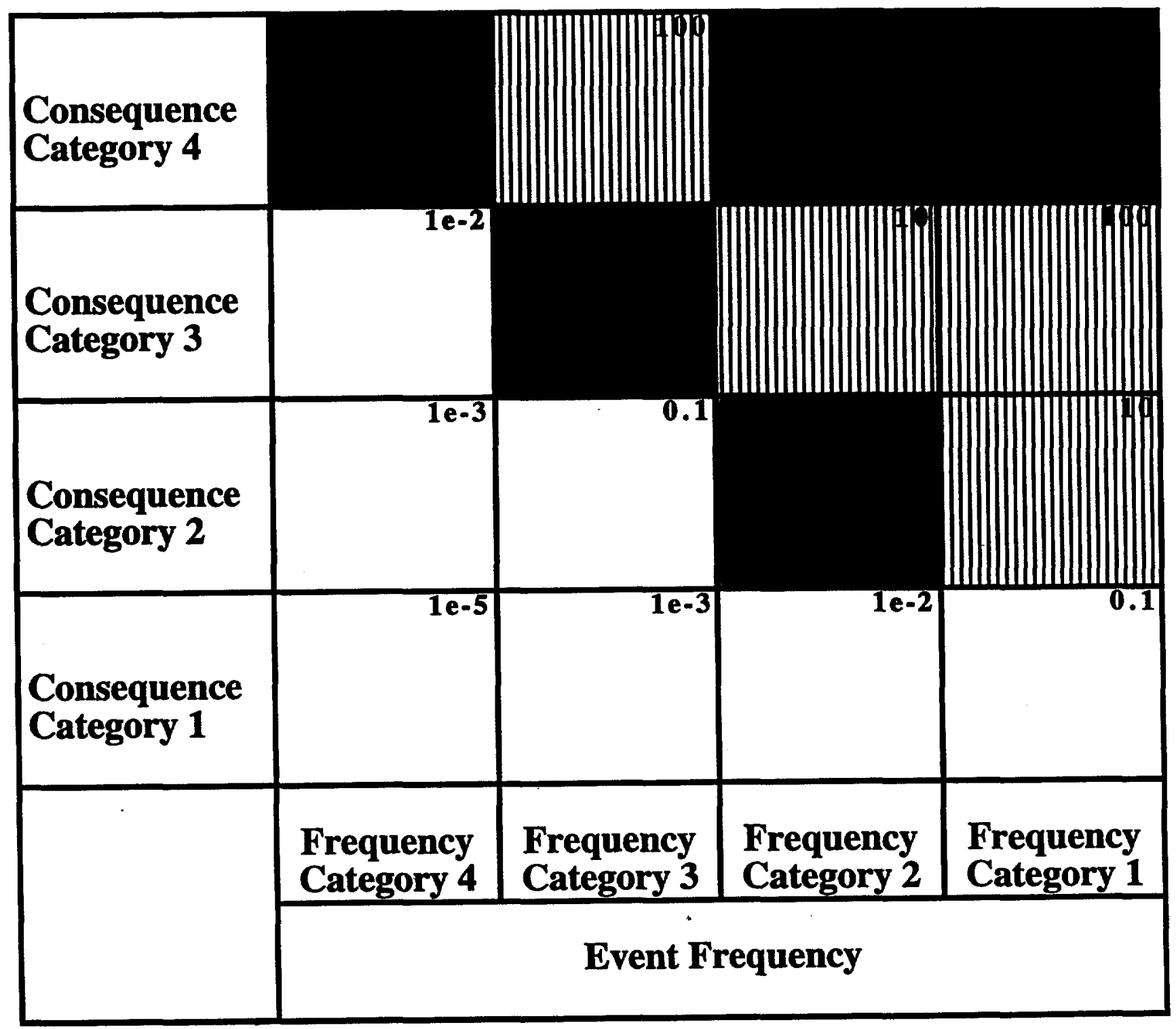

\begin{tabular}{|l|l|}
\hline & Risk Level 1 \\
\hline Risk Level 2 \\
\hline IIIIIIIIII Risk Level 3 \\
\hline Risk Level 4 \\
\hline
\end{tabular}

Figure 2: $\quad$ Risk Matrix for relative ranking of public chemical release events for the LDF Complex 


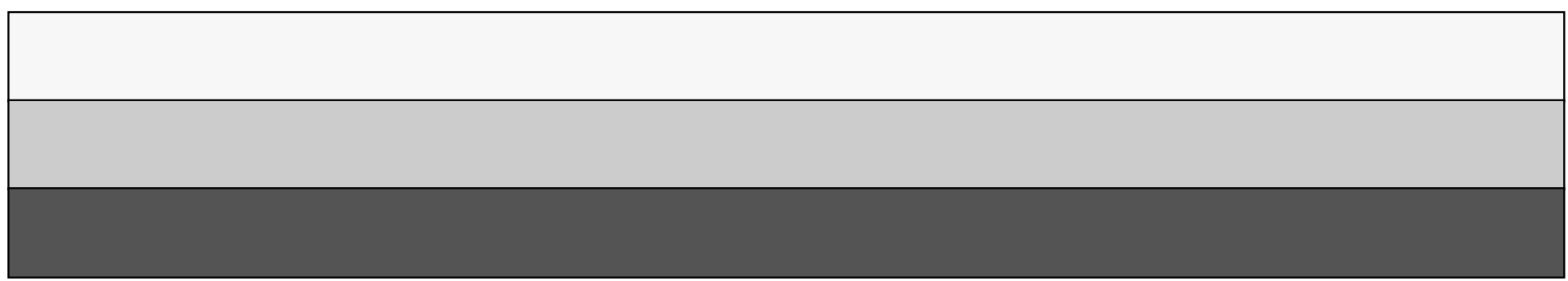

